\title{
Subthreshold Psychiatric Psychopathology in Functional Gastrointestinal Disorders: Can It Be the Bridge between Gastroenterology and Psychiatry?
}

\author{
Cristina Stasi, ${ }^{1}$ Cristiana Nisita, ${ }^{2}$ Sonia Cortopassi, ${ }^{2}$ Giorgio Corretti, ${ }^{3}$ Dario Gambaccini, ${ }^{4}$ \\ Nicola De Bortoli, ${ }^{4}$ Bernardo Fani, ${ }^{4}$ Natalia Simonetti, ${ }^{4}$ Angelo Ricchiuti, ${ }^{4}$ \\ Liliana Dell'Osso, ${ }^{2}$ Santino Marchi, ${ }^{4}$ and Massimo Bellini ${ }^{4}$ \\ ${ }^{1}$ Internal Medicine and Liver Unit, Department of Experimental and Clinical Medicine, Careggi University Hospital, Florence, Italy \\ ${ }^{2}$ Psychiatry Unit, Department of Clinical and Experimental Medicine, University of Pisa, Pisa, Italy \\ ${ }^{3}$ Functional Mental Health Unit of Adults-Northwest Tuscany Local Health Unit of Pisa, Pisa, Italy \\ ${ }^{4}$ Gastrointestinal Unit, Department of Clinical and Experimental Medicine, University of Pisa, Pisa, Italy \\ Correspondence should be addressed to Cristina Stasi; cristina.stasi@gmail.com
}

Received 1 August 2017; Revised 11 September 2017; Accepted 27 September 2017; Published 30 October 2017

Academic Editor: Per Hellström

Copyright (C) 2017 Cristina Stasi et al. This is an open access article distributed under the Creative Commons Attribution License, which permits unrestricted use, distribution, and reproduction in any medium, provided the original work is properly cited.

\begin{abstract}
Background and Aims. Functional gastrointestinal disorders (FGDs) are multifactorial disorders of the gut-brain interaction. This study investigated the prevalence of Axis I and spectrum disorders in patients with FGD and established the link between FGDs and psychopathological dimensions. Methods. A total of 135 consecutive patients with FGD were enrolled. The symptoms' severity was evaluated using questionnaires, while the psychiatric evaluation by clinical interviews established the presence/absence of mental (Diagnostic and Statistical Manual-4th edition, Axis I Diagnosis) or spectrum disorders. Results. Of the 135 patients, 42 (32.3\%) had functional dyspepsia, 52 (40.0\%) had irritable bowel syndrome, 21 (16.2\%) had functional bloating, and 20 (15.4\%) had functional constipation. At least one psychiatric disorder was present in $46.9 \%$ of the patients, while a suprathreshold panic spectrum was present in $26.2 \%$. Functional constipation was associated with depressive disorders $(p<0.05)$, while functional dyspepsia was related to the current major depressive episode $(p<0.05)$. Obsessive-compulsive spectrum was correlated with the presence of functional constipation and irritable bowel syndrome $(p<0.05)$. Conclusion. The high prevalence of subthreshold psychiatric symptomatology in patients with FGD, which is likely to influence the expression of gastrointestinal symptoms, suggested the usefulness of psychological evaluation in patients with FGDs.
\end{abstract}

\section{Introduction}

Functional gastrointestinal disorders (FGDs), better defined as disorders of gut-brain interaction, are a combination of various chronic or recurrent gastrointestinal symptoms without a structural basis to explain their clinical features [1]. These include different disorders and involve the entire digestive system. The symptoms of these disorders result from a complex interplay among different and synergic factors such as alterations of gut microbiota modulation and mucosal immunity, visceral hypersensitivity, and central nervous system dysregulation of the modulation of gut signalling and motor function [1-4]. Given their relevance, these factors should be encompassed in the relatively new field of neurogastroenterology [5].

It is worth noting that these factors are the most frequently reported conditions by the gastroenterologists and constitute an important portion of the general practitioner's work; they cause significant absenteeism from work, decrease the health-related quality of life, and increase the medical costs $[6,7]$.

The cross-cultural aspects and psychological factors in FGD are well recognized [8]. The psychological and environmental factors together with the psychological distress affect 
both the clinical expression and symptom severity and thus play a cocausative role in the onset and course of FGDs in susceptible persons [2, 3, 9-13].

Anxiety, depression, and somatoform disorders are often reported in patients with FGD, especially in those reporting to gastroenterology clinics and/or referral centres. However, this contrasts studies reporting patients with organic gastrointestinal problems who exhibit a lower prevalence of psychiatric disorders $[14,15]$.

Patients tend to consider their psychological problems as a consequence of the same cause that produces FGDs. However, in almost half of the patients with a psychiatric disorder, the psychopathological symptoms start before the gastrointestinal signs, while they begin at the same time in large portion of the remaining patients $[14,16]$.

Although many studies demonstrated a strong association between FGDs, psychological disturbances, and social stress [9], relatively few studies directly reported a relationship between FGDs and a well-defined psychiatric diagnosis.

Recently, Sahoo and Padhy [8] reviewed the crosscultural aspects and psychological factors in patients with IBS. They essentially indicated that carefully collecting the medical history and undertaking a holistic approach were relevant for the management of patients with IBS. Moreover, they outlined that a multidimensional approach, including both a psychiatric and gastroenterological assessment, is an essential part of an effective management of FGDs. In addition, on the basis of other multidisciplinary care models, a sharp decrease in health care resources should be expected because it could increase the efficacy and efficiency of the disease management, reaching a more rapid diagnosis, accelerating treatment planning, avoiding unnecessary duplication of tests, decreasing the patients' anxiety, and shortening the waiting times for receiving a coordinated plan of care [17].

Previously, the lack of reliable diagnostic criteria for classifying FGDs and mental disorders represented the most important methodological bias. In the last 20 years, the introduction of the DSM system and the Rome criteria strongly contributed to clarify better this intriguing matter.

Studies $[14,18-20]$ assessing the comorbidity of psychiatric symptoms and FGDs applied a traditional categorical diagnosis according to the different systems of classification of mental disorders (International Classification of Diseases [ICD-9], ICD-10, DSM-III-revised, and DSM-IV). Although these studies significantly contributed to the specification of this relationship, the categorical view of mental illness risks did not consider a large part of psychopathological signs and symptoms under the diagnostic threshold that may influence clinical management and prognosis of FGDs, such as other medical and psychiatric disorders.

The concept of spectrum disorders [21] has been developed in order to identify the whole amount of psychopathological signs and symptoms characterizing a mental disorder more efficiently. In addition to typical DSM core symptoms, the spectrum comprises isolated or atypical symptoms, often of low severity, as well as trait-behavioural features, which tend to persist, waxing and waning, through the lifespan. This use of prodromal, subthreshold, typical, atypical, residual, and trait-like symptoms allows a dimensional evaluation of anxiety and mood phenomena and a better comprehension of the psychopathological continuum [22-24].

Therefore, several assessment instruments, including structural interviews and self-report questionnaires, were developed and tested in recent years [25].

Based on these observations, the aims of this study were to investigate the prevalence of Axis I and spectrum disorders in a population of patients with FGD and to establish the possible links between some FGDs and some psychopathological dimensions.

\section{Patients and Methods}

From 1 February 2013 to 31 July 2014, 135 consecutive patients with FGD (34 men [25.2\%], 101 women [74.8\%]; mean age 43.22 \pm 15.05 years; age range 18-70 years), referred to the gastroenterology outpatient services of the Gastrointestinal Unit of the University of Pisa, Italy, were enrolled in this study.

The inclusion criteria were as follows: (1) patients referred for functional gastrointestinal disorders at the Gastroenterology Unit and (2) age range 18-70 years.

The following exclusion criteria were considered: (1) severe organic diseases; (2) significant changes in serum chemistry; (3) history of abdominal surgery (except appendectomy); (4) lactose intolerance (demonstrated by lactose breath test); (5) pregnancy; and (6) medication taken within $15 \mathrm{~d}$ prior to the study, which alters the autonomic response (e.g., anticholinergic drugs or beta blockers), psychotropic activity (e.g., tricyclic antidepressants, serotonin [5-HT] reuptake inhibitors, or benzodiazepines), and/or that potentially interfere with the gastrointestinal motility.

The study protocol was approved by the Ethical Committee of Pisa and was carried out in accordance with the tenets of the Helsinki Declaration (6th Revision, Seoul, 2008). Furthermore, written informed consent was obtained from each participant.

2.1. Gastroenterological Evaluation. The patients were grouped in accordance with their FGD: 42 (32.3\%) were diagnosed with functional dyspepsia (FD), 52 (40.0\%) with irritable bowel syndrome (IBS), 21 (16.2\%) with functional bloating (FB), and $20(15.4 \%)$ with functional constipation (FC).

The diagnosis of the different FGDs was performed in accordance with the Rome III criteria [26, 27].

The severity of the symptoms was evaluated using specific questionnaires including the IBS Symptom Severity Score (IBS-SSS) to evaluate abdominal symptom severity [28] and the Dyspepsia SSS [29]. Additionally, a "homemade" bowel habits questionnaire using a scale ranging from 0 (no symptoms) to 4 (symptoms present during $\geq 75 \%$ of bowel movements or days) [30] evaluated the frequency of (1) painful defecation, (2) manual manoeuvres facilitating defecation, (3) hard faeces, (4) watery faeces, (5) "fragmented" defecation, (6) sensation of anorectal blockage, (7) urge to defecate, (8) incontinence for 
gas and/or faeces, (9) abdominal pain, and (10) abdominal bloating.

The degree of interference of the gastrointestinal symptoms with the global well-being was expressed by a visual analogue scale $(0-100 \mathrm{~mm})$, with 0 indicating absent and 100 indicating unbearable.

2.2. Psychiatric Evaluation. The psychiatric evaluations were based on structured clinical interviews aimed to establish essentially the presence or absence of (1) mental disorders (DSM-IV Axis I diagnoses) and (2) spectrum disorder.

\subsection{Instruments}

2.3.1. Structured Clinical Interview for DSM-IV Patient Edition (SCID IV). The Structured Clinical Interview for DSM-IV Patient Edition (SCID IV) [31] was administered to diagnose Axis I disorders using the DSM-IV categorical criteria. SCID-IV is a hetero-evaluation instrument used to assess psychiatric Axis I disorder into different nosographic issues. The interview consists of a first part aimed to reveal the demographic data, such as work and educational level, as well as medical and pharmacological history. The second part consists of different sessions: mood disorders, episodes (session A), psychotic symptoms associated to mood disorders (session B), psychotic disorders (session C), mood disorders, course (session D), substance abuse disorders (session $\mathrm{E}$ ), anxiety disorders (session F), somatoform disorders (session G), eating disorders (session $\mathrm{H}$ ), and adjustment disorders (session I).

2.3.2. Structured Clinical Interview for Panic-Agoraphobic Spectrum-Self-Report Version (SCI PAS-sr). SCI-PAS [32] is a clinical interview aimed to evaluate the presence or absence of panic and agoraphobic spectrum symptomatology. It consists of 114 items grouped into 8 domains: (1) separation sensitivity; (2) panic-like symptoms (typical and atypical); (3) stress sensitivity; (4) substance and medication sensitivity; (5) anxious expectation; (6) agoraphobia; (7) illness-related phobia; and (8) reassurance orientation. Every item was coded as "true" (1) or "false" (0), and the diagnostic threshold was 35 .

2.3.3. Structured Clinical Interview for Mood Spectrum-SelfReport Version (SCI MOODS-sr). SCI-MOOD [33] consists of 140 dichotomous items aimed to evaluate the presence/ absence of mood spectrum signs and symptoms and grouped into seven domains: (1) mood depressed; (2) mood manic; (3) energy depressed; (4) energy manic; (5) cognition depressed; (6) cognition manic; and (7) rhythmicity and vegetative functions. The diagnostic threshold was 61 .

2.3.4. Structured Clinical Interview for Anorexic-Bulimic Spectrum-Self-Report Version (SCI ABS-sr). SCI ABS-sr [34] is a clinical interview aimed to evaluate the presence or absence of anorexic-bulimic spectrum symptomatology. It consists of 134 items grouped into nine domains: (1) attitudes and beliefs; (2) history of weight loss; (3) self-esteem and satisfaction; (4) phobias; (5) avoidance and compulsive behaviours; (6) weight maintenance; (7) eating disorders;
(8) associated features and consequences; and (9) impairment and insight. The diagnostic threshold was 45 points where the answer coded by yes was equal to 1 .

2.3.5. Structured Clinical Interview for Obsessive-Compulsive Spectrum-Self-Report Version (SCI OBS-sr). SCI OBS [35] is a clinical interview aimed to evaluate the presence/absence of obsessive-compulsive spectrum symptoms. The SCI-OBS consists of 196 items grouped into seven domains: (1) childhood/adolescence experiences; (2) doubt; (3) hypercontrol; (4) attitudes toward time; (5) perfectionism; (6) repetition and automation; and (7) specific themes. Every item was coded as present or absent, and the diagnostic threshold was 59 .

2.3.6. Structured Clinical Interview for Social Anxiety Spectrum-Self-Report Version (SCI SHY-sr). SCI SHY [35] is a clinical interview aimed to evaluate the presence/absence of social anxiety spectrum symptoms and consists of 164 items grouped into four domains: (1) social phobic traits during childhood and adolescence; (2) interpersonal sensitivity; (3) behavioural inhibition and somatic symptoms; and (4) specific anxiety and phobic features. The appendix explores the use of psychoactive substances, which is a frequent complication of the social anxiety disorder. The diagnostic threshold was 59.

2.3.7. Statistical Analysis. All data were analysed using SPSS (SPSS Inc., Chicago, IL, USA) and Stata 12 software (Stata Statistical Software, College Station, TX, USA). All variables are expressed as the mean \pm standard deviation. The numerical comparison of the continuous data was performed using the $t$-test for paired samples. Statistical significance was set at a value of $p<0.05$. The Chi-square test was used to evaluate the categorical variables. Linear regression analysis between two variables was performed using Pearson correlation.

We have performed the multinomial logistic regression analysis to evaluate the associations between the global well-being and spectrum disorders/Axis I diagnosis, the well-being in IBS patients and spectrum disorders/Axis I diagnosis, the well-being in FB patients and spectrum disorders/Axis I diagnosis, the well-being in FD patients and spectrum disorders/Axis I diagnosis, and the well-being in FC patients and spectrum disorders/Axis I diagnosis. In this analysis, the score $\geq 40$ was considered interfering with well-being.

\section{Results}

Table 1 summarizes the functional gastrointestinal diagnosis groups of patients with FDGs.

At least one diagnosis of the psychiatric Axis I disorder was present in $46.9 \%$ of the patients. In particular, the generalised anxiety disorder was diagnosed in $20.0 \%$ of the patients, while panic disorders, including panic disorder, panic without agoraphobia, and agoraphobia without panic attack, were diagnosed in $27.7 \%$ of the patients. Mood disorders were the most frequent and were diagnosed in $13.1 \%$ of the patients (Table 2). 
TABLe 1: Distribution $(N, \%)$ of different FGDs studied.

\begin{tabular}{lcc}
\hline Functional gastrointestinal disorders & $(N: 135)$ & $\%$ \\
\hline Functional constipation & 20 & 15.4 \\
Irritable bowel syndrome & 52 & 40.0 \\
Functional dyspepsia & 42 & 32.3 \\
Functional bloating & 21 & 16.2 \\
\hline
\end{tabular}

Regarding spectrum symptomatology, a suprathreshold panic spectrum was present in $26.2 \%$ of the patients. Despite the low prevalence of Axis I obsessive-compulsive disorder $(0.8 \%)$ and social phobia (1.5\%), a high percentage of the patients exhibited a positive obsessive (19.2\%) and social phobic (23.8\%) spectrum. Of the entire population, $39.2 \%$ had at least one spectrum symptomatology of anxiety, while $43.1 \%$ had at least one spectrum symptomatology of affective disorders.

A low prevalence (3.9\%) of axis eating disorders (anorexia, bulimia, and binge eating disorder) and a higher (15.4\%) suprathreshold spectrum symptomatology were also observed.

Almost $13.1 \%$ of the patients had an Axis I mood disorder, while $20.8 \%$ had a positive mood spectrum (Table 2 ).

Table 3 summarizes the prevalence of psychiatric disorders and spectrum symptomatology in the different FGDs. Significant associations were observed between FGDs (in particular FC) and Axis I diagnosis depressive disorders (dysthymia and recurrent depression; $p<0.05$ ), while current major depressive episode was significantly related to $\mathrm{FD}(p<0.05)$.

When we considered the relationship between FGDs and spectrum disorders, obsessive-compulsive spectrum was significantly related to the presence of FC and IBS $(p<0.05)$.

Considering the severity of the gastrointestinal symptoms and the degree of interference with the global well-being of the patients, a social phobic spectrum showed a significant correlation with IBS $(p<0.05)$. Indeed, in the IBS group, the comorbidity with social phobia was related to a lower level of well-being, particularly with symptoms such as defecation urgency $(p<0.01)$ and abdominal pain relieved by evacuation $(p<0.05)$. However, the presence of spectrum disorders was positively related to the degree of interference of the gastrointestinal symptoms with the subjective global well-being. Thus, patients without an Axis I disorder or a suprathreshold spectrum had a significantly lower level of interference with the global well-being $(p<0.05)$.

Moreover, the presence of obsessive, social phobic, and eating positive spectrums significantly decreased the global well-being score. Obsessive and eating spectrums were directly related to the well-being impairment of the IBS group, while the social phobic spectrum was related to the impairment of the well-being of the FC and FB groups. A positive mood spectrum was inversely related to the wellbeing of the patients with IBS (Tables 4 and 5).

When we performed the multinomial logistic regression analysis, we found a significant association between the global well-being and spectrum disorders $(p=0.01)$, but we found no significant association between global well-being and Axis I diagnosis $(p=0.26)$. When we considered the different FGD groups, we found a significant association between well-being in FC patients and spectrum disorders $(p=0.03)$.

\section{Discussion}

The relationship between FGDs and psychiatric disorders has been widely studied, but the results have been often conflicting, mainly because of the complexity of the question and the methodological bias in many studies.

Our study fully reflected the disputes concerning the intriguing question "irritable brain or irritable bowel." The results confirmed a high psychiatric comorbidity in FGDs, particularly mood and anxiety disorders, in the sense of full diagnosis (one-third of the sample met the criteria for the Axis I mental disorder).

However, independently of the full-blown diagnosis, a particular value of this study was the organisation for analysis, using appropriate standardized instruments, of a psychopathological dimension, often labelled as "vulnerability psychological features" and observed initially by gastroenterologists.

For the first time, this study analysed the subthreshold psychiatric symptomatology in patients with different FGDs. It also confirmed the previous observations about the high prevalence of Axis I psychiatric disorders among patients with FGD $[3,4,9,17,30,36]$, particularly anxiety and mood disorders. Subthreshold psychiatric symptomatology is formed by a variety of symptoms that do not conform to a formal diagnosis of mental disorder according to standard psychiatric classification, but it impacts significantly in quality of life.

However, the presence of a psychiatric Axis I disorder seemed to explain partly the complex relationship between the brain and the gut, particularly the clinical expressivity of the functional gastrointestinal disorders. In previous studies $[3,4,36]$, investigating psychological disorders, we found a high prevalence of psychological disorders, but we have also found that many patients had elevated scores for psychopathology, however without reaching a significant score for diagnosis. This led us to investigate spectrum disorders in patients with FGDs. The comorbidity between FGDs and psychiatric disorders is not simply restricted to Axis I disorders but it also widely extends to spectrum psychopathology, such as subclinical, prodromal, or residual symptoms. Spectra instruments permit the collection of underlying soft psychopathological symptoms generally defined as neurotic traits or stress-related symptoms. Spectrum diagnoses showed a strong coherence with DSM-IV diagnoses and provided additional clinical information. It is likely that this assessment can be used in patients with nonpsychiatric disorders to provide a more comprehensive picture of symptoms and behavioural traits. Additionally, previous studies indicated that the presence of spectrum symptomatology has a clinical relevant significance as a predictor of outcome in major depressive and bipolar disorders [37].

In this study, the use of the spectrum instruments has enabled pointing out to the presence of a subthreshold 
TABLE 2: Axis I and mood spectrum disorders in patients with functional gastrointestinal disorders.

\begin{tabular}{|c|c|c|c|c|c|}
\hline Axis I diagnosis & $(N)$ & $\%$ & Spectrum & Mean + SD & Overthreshold \\
\hline \multicolumn{6}{|l|}{ Anxiety disorders } \\
\hline Panic & 24 & 18.5 & \multirow{6}{*}{ SCI-PAS } & \multirow{6}{*}{$25.99 \pm 17.54$} & \multirow{6}{*}{$26.2(34)$} \\
\hline Panic with agoraphobia & 10 & 7.7 & & & \\
\hline Agoraphobia without panic attack & 2 & 1.5 & & & \\
\hline Single phobia & 4 & 3.1 & & & \\
\hline Anxiety NAS & 4 & 3.1 & & & \\
\hline GAD & 26 & 20.0 & & & \\
\hline Social phobia & 2 & 1.5 & SCI-SHY & $39.47 \pm 31.07$ & $23.8(31)$ \\
\hline OCD & 1 & 0.8 & SCI-OBS & $39.64 \pm 24.69$ & $19.2(25)$ \\
\hline Somatoform disorders & 2 & 1.5 & & & \\
\hline \multicolumn{6}{|l|}{ Mood disorders } \\
\hline Past major depressive episode & 13 & 10.0 & SCI-MOODS & $38.25 \pm 24.45$ & \multirow{5}{*}{$20.8(27)$} \\
\hline Current major depressive episode & 2 & 1.5 & & & \\
\hline Recurrent depression & 1 & 0.8 & Dep & $24.78 \pm 17.24$ & \\
\hline Dysthymia & 1 & 0.8 & Man & $13.48 \pm 9.55$ & \\
\hline Somatization disorders & 1 & 0.8 & & & \\
\hline \multicolumn{6}{|l|}{ Eating disorders } \\
\hline Anorexia & 1 & 0.8 & & & \\
\hline Bulimia & 4 & 3.1 & SCI-ABS & $20.53 \pm 19.66$ & $15.4(20)$ \\
\hline BED & 0 & 0.0 & & & \\
\hline
\end{tabular}

SCI-PAS: Structured Clinical Interview for Panic-Agoraphobic Spectrum-self-report version; SCI-SHY: Structured Clinical Interview for Social Anxiety Spectrum; SCI-OBS: Structured Clinical Interview for Obsessive-Compulsive Spectrum; SCI-MOODS: Structured Clinical Interview for Mood Spectrum; SCI-ABS: Structured Clinical Interview for Anorexic-Bulimic Spectrum.

psychopathology in $44.6 \%$ of the study sample, compared with an almost overlapping percentage of diagnosis of Axis I (46.9\%). It is worth noting that a spectrum diagnosis was also present in $27.5 \%$ of patients with no major psychiatric disorder; therefore, these spectrum instruments are also very useful in patients with nonpsychiatric disorders not affected by Axis I disorders, for whom a traditional psychiatric approach is not feasible.

The distribution of spectrum diagnoses in our sample (PAS 26.2\%, MOOD 20.8\%, OBS 19.2\%, SHY 23.8\%, and ABS 15.4\%) demonstrated an affective continuum that characterized the dimensional psychopathological profile of this cohort with clinical nonpsychiatric disorders. Our data further indicated a close relationship between FGDs and anxiety spectrum symptomatology, such as that underlined by the high prevalence of panic-agoraphobic, social phobic, and obsessive-compulsive spectrums. In consideration of this, the unspecific terms of "neuroticism" and "stress reaction" often used to label patients with FGDs in the past may be replaced by the term "anxious spectra."

However, the low-grade inflammation associated with the release of interleukin- (IL-) 1, IL-6, and tumour necrosis factor- (TNF-) $\alpha$ may activate the cerebral circuits via afferent fibres [2]. Indeed, recent findings demonstrated that the gut microbiota plays a pivotal role in stress-related psychiatric disorders $[38,39]$ and that the sensitivity and gastrointestinal motility are both regulated by the central and peripheral nervous systems via the complex interplay between the brain and gut $[3,4]$. 5-HT plays a key role in the regulation of visceral pain and in the secretion and initiation of the peristaltic reflex. However, altered levels of 5-HT are also detected in many different psychiatric disorders such as anxiety, depression, obsessive-compulsive disorders, and phobia $[13,17]$. The $5-$ HT released in the gut from the enterochromaffin cells regulates the sensory, motor, and secretory functions of the digestive system through interactions with intrinsic and extrinsic nervous pathways. Intrinsic innervation to the gut is supplied by neurons of the ENS, including the myenteric and submucosal plexus. Extrinsic innervation is provided by the autonomic nervous system (both sympathetic and parasympathetic) and is arranged to function in a bidirectional manner $[11,40,41]$. Moreover, the 5-HT concentrations are regulated by its reuptake, which is operated by the 5 -HT transporter (SERT) expressed both in neurons and intestinal epithelium [40-43].

Our data demonstrated that the presence of spectrum diagnoses influences the clinical picture of FGDs, particularly well-being, quality of life, and severity of the FGD symptoms.

Spectrum diagnoses tend to be related to a low well-being referred by patients and to a higher severity of FGD symptoms. In fact, the presence of spectrum disorders, in particular in FC patients after multivariate analysis, was positively associated with the degree of interference of the gastrointestinal symptoms with the subjective global well-being. This could indicate that psychic dimension may change the perception of the symptoms.

This is possibly owing to the psychological disorders that in turn lead to a chronic state of amplification of symptoms, 
TABLE 3: The prevalence of psychiatric disorders and spectrum symptomatology in the different FGDs.

\begin{tabular}{|c|c|c|c|c|}
\hline & $\mathrm{FC} \%(N=20)$ & IBS \% $(N=52)$ & $\mathrm{FD} \%(N=42)$ & $\mathrm{FB} \%(N=21)$ \\
\hline At least 1 Axis I disorder & $60.0(12)$ & $36.5(19)$ & $52.4(22)$ & $47.6(10)$ \\
\hline Panic & $25.0(5)$ & $17.3(9)$ & $19.0(8)$ & $14.3(3)$ \\
\hline Panic with agoraphobia & $15.0(3)$ & $3.8(2)$ & $9.5(4)$ & $4.8(1)$ \\
\hline Agoraphobia without panic & $5.0(1)$ & $0.0(0)$ & $2.4(1)$ & $0.0(0)$ \\
\hline Single phobia & $5.0(1)$ & $1.9(1)$ & $2.4(1)$ & $4.8(1)$ \\
\hline Anxiety NAS & $5.0(1)$ & $1.9(1)$ & $0.0(0)$ & $9.5(2)$ \\
\hline GAD & $20.0(4)$ & $17.3(9)$ & $26.2(11)$ & $14.3(3)$ \\
\hline Social phobia & $5.0(1)$ & $1.9(1)$ & $0.0(0)$ & $0.0(0)$ \\
\hline OCD & $0.0(0)$ & $0.0(0)$ & $2.4(0)$ & $0.0(0)$ \\
\hline Somatoform disorders & $5.0(1)$ & $1.9(1)$ & $0.0(0)$ & $0.0(0)$ \\
\hline Past MDE & $9.5(2)$ & $11.5(6)$ & $9.5(4)$ & $14.3(3)$ \\
\hline Current MDE & $0.0(0)$ & $0.0(0)$ & $4.8(2)$ & $0.0(0)$ \\
\hline Recurrent depression & $5.0(1)$ & $0.0(0)$ & $0.0(0)$ & $0.0(0)$ \\
\hline Dysthymia & $5.0(1)$ & $0.0(0)$ & $0.0(0)$ & $0.0(0)$ \\
\hline Somatization disorders & $0.0(0)$ & $0.0(0)$ & $2.4(1)$ & $0.0(0)$ \\
\hline Anorexia & $0.0(0)$ & $0.0(0)$ & $2.4(1)$ & $0.0(0)$ \\
\hline Bulimia & $5.0(1)$ & $1.9(1)$ & $2.4(1)$ & $4.8(1)$ \\
\hline BED & $0.0(0)$ & $0.0(0)$ & $0.0(0)$ & $0.0(0)$ \\
\hline \multicolumn{5}{|l|}{ Spectrum symptomatology } \\
\hline Overthreshold SCI-OBS & $35.0(7)$ & $9.6(5)$ & $19.0(8)$ & $23.8(5)$ \\
\hline Overthreshold SCI-SHY & $25.0(5)$ & $21.2(11)$ & $31.0(13)$ & $14.3(3)$ \\
\hline Overthreshold SCI-PAS & $30.0(6)$ & $21.2(11)$ & $28.6(12)$ & $23.8(5)$ \\
\hline At least 1 anxiety spectrum & $50.0(10)$ & $32.7(17)$ & $42.9(18)$ & $33.3(7)$ \\
\hline Overthreshold SCI-ABS & $25.0(5)$ & $9.6(5)$ & $16.7(7)$ & $14.3(3)$ \\
\hline Overthreshold SCI-MOODS & $35.0(7)$ & $13.5(7)$ & $28.8(10)$ & $14.3(3)$ \\
\hline
\end{tabular}

NAS: anxiety in autistic adults; GAD: generalized anxiety disorder; OCD: obsessive-compulsive disorder; MDE: mood disorder event; BED: bipolar disorder event; SCI-PAS: Structured Clinical Interview for Panic-Agoraphobic Spectrum-self-report version; SCI-SHY: Structured Clinical Interview for Social Anxiety Spectrum; SCI-OBS: Structured Clinical Interview for Obsessive-Compulsive Spectrum; SCI-MOODS: Structured Clinical Interview for Mood Spectrum; SCI-ABS: Structured Clinical Interview for Anorexic-Bulimic Spectrum; FC: functional constipation; IBS: irritable bowel syndrome; FD: functional dyspepsia; FB: functional bloating.

TABLE 4: Differences in FDGs between nonpsychiatric patients and patients with the presence of Axis I and/or spectrum disorders.

\begin{tabular}{lccc}
\hline & Healthy & $\begin{array}{c}\text { Axis I and/or } \\
\text { spectrum disorders }\end{array}$ & $p$ \\
\hline Global well-being & 36.70 & 31.50 & $<0.05$ \\
IBS well-being & 17.40 & 8.75 & $<0.01$ \\
FB well-being & 5.70 & 4.19 & $\mathrm{~ns}$ \\
Dyspepsia well-being & 10.90 & 12.06 & $\mathrm{~ns}$ \\
FC well-being & 2.70 & 6.50 & $\mathrm{~ns}$ \\
\hline
\end{tabular}

which originates either at the level of the CNS (hypervigilance on physical perception) or at the visceral level (hypersensitivity and hypermotility) [2].

Obsession, which is revealed by a positive obsessivecompulsive spectrum, was correlated with FGDs such as FC and IBS.

Interesting conclusions could be inferred from the second part of the analysis regarding the spectrum psychopathology, severity, and global well-being both in the whole
TABLE 5: Difference between psychiatric disorders (DSM IV) versus spectrum diagnosis stratified for well-being.

\begin{tabular}{lccccc}
\hline & OBS & SHY & PAS & MOODS & ABS \\
\hline Global well-being & $26.80^{* *}$ & $27.58^{* *}$ & 32.79 & 29.07 & $27.25^{*}$ \\
IBS well-being & $4.60^{* *}$ & 10.32 & 8.24 & $6.30^{*}$ & $4.00^{* *}$ \\
FB well-being & 5.20 & $1.61^{*}$ & 2.50 & 4.63 & 2.50 \\
FD well-being & 11.20 & 13.70 & 13.82 & 11.85 & 13.50 \\
FC well-being & 5.80 & $1.94^{*}$ & 8.23 & 6.30 & 7.25 \\
${ }^{*} p<0.05 ;{ }^{* *} p<0.01$. & & & & &
\end{tabular}

population and in the FGD groups. Higher gastroenterological symptomatic scores with a social phobic spectrum were detected in patients with IBS compared to the other FGD groups. Social phobic psychopathological nucleus is based on the overestimation of judgement and criticism from others; it may be reflected by an increase of frequency and severity of anxiety-mediated IBS symptoms. The correlation with defecation urgency and pain relief after evacuation 
seems to confirm this view. Indeed, the fear of decontrol in the sphincter functions, which significantly increases the defecation urgency, and the significant decrease in anxiety levels after evacuation could be perceived by the patients as a reduction in the pain and urgency.

The obsessive and social-phobic dimensions were shown to be associated with "poor well-being"; thus, with a worse quality of life, they were assessed as subjective satisfaction and interference. However, PAS, MOOD, and ABS spectrum diagnoses as well as Axis I of panic attack disorder (DAP) generalised anxiety disorder and major depression; although they were easier to collect in nonpsychiatric clinical practice, they do not show equal predictive powers.

In particular, the well-being of patients with FGDs was significantly impaired when the gastrointestinal disease was associated with a positive obsessive and social phobic spectrum.

Obsessive patients seemed to be highly limited in their functioning by this reactivity and instability in evacuation, thereby triggering an inhibitory behaviour and a reduction in the quality of life. The association between low well-being in patients with $\mathrm{FB}$ and social phobic spectrum underlines the inhibited behaviour due to the fear of emitting intestinal sounds in public.

Especially spectrum diagnosis interfered negatively with the global well-being of the patients affected by FGDs, regardless of the specific FGD type and the severity of the gastrointestinal symptoms.

Subjective satisfaction, quality of life, and global wellbeing are variables that may increase the level of sufferance referred to a symptom, and they could also inspire the patients to refer to a physician or a gastroenterologist.

\section{Conclusions}

In conclusion, the high presence of subthreshold psychiatric symptomatology in patients with FGDs suggested the usefulness of psychological evaluations in these patients, in whom probably a brief self-evaluation scale could be used in the clinical gastroenterological practice, targeted to reveal subclinical psychopathological features that are likely to influence significantly the expression of the gastrointestinal symptoms. The second step could be represented by the evaluation of Axis I psychiatric disorders and the global level of well-being. Further studies are needed to clarify further the pathophysiology of psychological disorders often associated with FGDs.

\section{Conflicts of Interest}

The authors declare that they have no conflicts of interest.

\section{References}

[1] D. A. Drossman and W. L. Hasler, "Rome IV-functional GI disorders: disorders of gut-brain interaction," Gastroenterology, vol. 150, no. 6, pp. 1257-1261, 2016.

[2] C. Stasi, M. Rosselli, M. Bellini, G. Laffi, and S. Milani, "Altered neuro-endocrine-immune pathways in the irritable bowel syndrome: the top-down and the bottom-up model," Journal of Gastroenterology, vol. 47, no. 11, pp. 1177-1185, 2012.

[3] C. Stasi, M. Bellini, F. Costa et al., "Neuroendocrine markers and psychological features in patients with irritable bowel syndrome," International Journal of Colorectal Disease, vol. 28, no. 9, pp. 1203-1208, 2013.

[4] C. Stasi, M. Bellini, D. Gambaccini et al., "Neuroendocrine dysregulation in irritable bowel syndrome patients: a pilot study," Journal of Neurogastroenterology and Motility, vol. 23, no. 3, pp. 428-434, 2017.

[5] P. J. Pasricha, "Neurogastroenterology: a great career choice for aspiring gastroenterologists thinking about the future," Gastroenterology, vol. 140, no. 4, pp. 1126-1128, 2011.

[6] D. A. Drossman, Z. Li, E. Andruzzi et al., "U.S. householder survey of functional gastrointestinal disorders. Prevalence, sociodemography and health impact," Digestive Diseases and Sciences, vol. 38, no. 9, pp. 1569-1580, 1993.

[7] A. F. Peery, S. D. Crockett, A. S. Barritt et al., "Burden of gastrointestinal, liver, and pancreatic diseases in the United States," Gastroenterology, vol. 149, no. 7, pp. 1731-1741e3, 2015.

[8] S. Sahoo and S. K. Padhy, "Cross-cultural and psychological issues in irritable bowel syndrome," Journal of Gastroenterology and Hepatology, vol. 32, no. 10, pp. 1679-1685, 2017.

[9] R. Lea and P. J. Whorwell, "New insights into the psychosocial aspects of irritable bowel syndrome," Current Gastroeneterology Reports, vol. 5, pp. 343-350, 2003.

[10] M. Camilleri and A. C. Ford, "Irritable bowel syndrome: pathophysiology and current therapeutic approaches," Handbook of Experimental Pharmacology, vol. 239, pp. 75-113, 2017.

[11] C. Stasi, M. Rosselli, A. L. Zignego, G. Laffi, and S. Milani, "Serotonin and its implication in the side-effects of interferon-based treatment of patients with chronic viral hepatitis: pharmacological interventions," Hepatology Research, vol. 44, no. 1, pp. 9-16, 2014.

[12] M. D. Gershon, "Serotonin and its implication for the management of irritable bowel syndrome," Reviews in Gastroenterological Disorders, vol. 3, Supplement 2, pp. S25-S34, 2003.

[13] M. D. Crowell, M. P. Jones, L. A. Harris, T. N. Dineen, V. A. Schettler, and K. W. Olden, "Antidepressants in the treatment of irritable bowel syndrome and visceral pain syndromes," Current Opinion in Investigational Drugs, vol. 5, pp. 736742, 2004

[14] R. B. Lydiard, M. D. Fossey, W. Marsh, and J. C. Ballenger, "Prevalence of psychiatric disorders in patients with irritable bowel syndrome," Psychosomatics, vol. 34, no. 3, pp. 229234, 1993.

[15] D. A. Drossman, "The functional gastrointestinal disorders and the Rome II process," Gut, vol. 45, Supplement 2, pp. II1-II5, 1999.

[16] F. H. Creed, "Psychopathology of functional disorder of the gut," in Functional Disorders of the Gut: A Handbook for Clinicians, S. F. Phillips and D. L. Wingate, Eds., WB Saunders Co., London, 1998.

[17] M. Bellini, D. Gambaccini, and G. Bassotti, "Comorbidities in functional gastrointestinal diseases: do we need a lone ranger or a dream team?," Digestive and Liver Disease, vol. 48, no. 5, pp. 562-564, 2016.

[18] D. A. Drossman, M. Camilleri, E. A. Mayer, and W. E. Whitehead, "AGA technical review on irritable syndrome," Gastroenterology, vol. 123, no. 6, pp. 2108-2131, 2002. 
[19] J. Kruimel, C. Leue, B. Winkens et al., "Integrated medicalpsychiatric outpatient care in functional gastrointestinal disorders improves outcome: a pilot study," European Journal of Gastroenterology \& Hepatology, vol. 27, no. 6, pp. 721-727, 2015.

[20] M. Fadgyas-Stanculete, A. M. Buga, A. Popa-Wagner, and D. L. Dumitrascu, "The relationship between irritable bowel syndrome and psychiatric disorders: from molecular changes to clinical manifestations," Journal of Molecular Psychiatry, vol. 2, no. 1, p. 4, 2014.

[21] G. B. Cassano, S. Michelini, M. K. Shear, E. Coli, J. D. Maser, and E. Frank, "The panic-agoraphobic spectrum: a descriptive approach to the assessment and treatment of subtle symptoms," The American Journal of Psychiatry, vol. 154, no. 6, pp. 27-38, 1997.

[22] C. D. Sherbourne, K. B. Wells, R. D. Hays, W. Rogers, M. A. Burnam, and L. L. Judd, "Subthreshold depression and depressive disorder: clinical characteristics of general medical and mental health specialty outpatients," The American Journal of Psychiatry, vol. 151, no. 12, pp. 1777-1784, 1994.

[23] H. S. Akiskal, L. L. Judd, J. C. Gillin, and H. Lemmi, "Subthreshold depressions: clinical and polysomnographic validation of dysthymic, residual and masked forms," Journal of Affective Disorders, vol. 45, no. 1-2, pp. 53-63, 1997.

[24] W. Coryell, "Do psychotic, minor and intermittent depressive disorders exist on a continuum?," Journal of Affective Disorders, vol. 45, no. 1-2, pp. 75-83, 1997.

[25] L. Dell'Osso, C. Carmassi, P. Rucci et al., "A multidimensional spectrum approach to post-traumatic stress disorder: comparison between the structured clinical interview for trauma and loss spectrum (SCI-TALS) and the self-report instrument (TALS-SR)," Comprehensive Psychiatry, vol. 50, no. 5, pp. 485-490, 2009.

[26] J. Tack, N. J. Talley, M. Camilleri et al., "Functional gastroduodenal disorders," Gastroenterology, vol. 130, no. 5, pp. 14661479, 2006.

[27] G. F. Longstreth, W. G. Thompson, W. D. Chey, L. A. Houghton, F. Mearin, and R. C. Spiller, "Functional bowel disorders," Gastroenterology, vol. 130, no. 5, pp. 1480-1491, 2006.

[28] C. Y. Francis, J. Morris, and P. J. Whorwell, "The irritable bowel severity scoring system: a simple method of monitoring irritable bowel syndrome and its progress," Alimentary Pharmacology and Therapeutics, vol. 11, no. 2, pp. 395-402, 1997.

[29] V. Stanghellini, C. Tosetti, G. Barbara, B. Salvioli, R. De Giorgio, and R. Corinaldesi, "Management of dyspeptic patients by general practitioners and specialists," Gut, vol. 43, Supplement 1, pp. S21-S23, 1998.

[30] M. Bellini, D. Gambaccini, L. Bazzichi et al., "Bioelectrical impedance vector analysis in patients with irritable bowel syndrome on a low FODMAP diet: a pilot study," Techniques in Coloproctology, vol. 21, no. 6, pp. 451-459, 2017.

[31] M. B. First, R. L. Spitzer, M. Gibbon, and J. B. Williams, Structured Clinical Interview for the DSM-IV Axis I Disorders, American Psychiatric Press, Inc., Washington, DC, USA, 1996.

[32] G. B. Cassano, S. Banti, M. Mauri et al., "Internal consistency and discriminant validity of the structured clinical interview for panic agoraphobic spectrum (SCI-PAS)," International Journal of Methods in Psychiatric Research, vol. 8, pp. 138-145, 1999.
[33] A. Fagiolini, L. Dell'Osso, S. Pini et al., "Validity and reliability of a new instrument for assessing mood symptomatology: the structured clinical interview for mood spectrum (SCIMOODS)," International Journal of Methods in Psychiatric Research, vol. 8, pp. 71-81, 1999.

[34] M. Mauri, C. Borri, S. Baldassari et al., "Acceptability and psychometric properties of the structured clinical interview for anorexic-bulimic spectrum (SCI-ABS)," International Journal of Methods in Psychiatric Research, vol. 9, pp. 68-78, 2000.

[35] L. Dell'Osso, G. B. Cassano, N. Sarno et al., "Validity and reliability of the structured clinical interview for obsessivecompulsive spectrum (SCI-OBS) and of the structured clinical interview for social phobia spectrum (SCI-SHY)," International Journal of Methods in Psychiatric Research, vol. 9, pp. 11-24, 2000.

[36] M. Bellini, L. Rappelli, P. Alduini et al., "Pelvic floor dyssynergia and psychiatric disorders. Does the snake bite its tail?," Minerva Gastroenterologica e Dietologica, vol. 49, no. 2, pp. 135-139, 2003.

[37] M. K. Shear, E. Frank, P. Rucci et al., "Panic-agoraphobic spectrum: reliability and validity of assessment instruments," Journal of Psychiatric Research, vol. 35, no. 1, pp. 59-66, 2001.

[38] J. R. Kelly, G. Clarke, J. F. Cryan, and T. G. Dinan, "Brain-gutmicrobiota axis: challenges for translation in psychiatry," Annals of Epidemiology, vol. 26, no. 5, pp. 366-372, 2016.

[39] M. Pigrau, B. K. Rodiño-Janeiro, M. Casado-Bedmar et al., "The joint power of sex and stress to modulate brain-gutmicrobiota axis and intestinal barrier homeostasis: implications for irritable bowel syndrome," Neurogastroenterology \& Motility, vol. 28, no. 4, pp. 463-486, 2016.

[40] D. Y. Kim and M. Camilleri, "Serotonin: a mediator of the brain-gut connection," The American Journal of Gastroenterology, vol. 95, no. 10, pp. 2698-2709, 2000.

[41] M. D. Gershon, "Plasticity in serotonin control mechanisms in the gut," Current Opinion in Pharmacology, vol. 3, pp. 600-607, 2003.

[42] J.-X. Chen, H. Pan, T. P. Rothman, P. R. Wade, and M. D. Gershon, "Guinea pig 5-HT transporter: cloning, expression, distribution, and function in intestinal sensory reception," American Journal of Physiology - Gastrointestinal and Liver Physiology, vol. 275, no. 3, Part 1, pp. G433-G448, 1998.

[43] R. Colucci, D. Gambaccini, N. Ghisu et al., "Influence of the serotonin transporter 5HTTLPR polymorphism on symptom severity in irritable bowel syndrome," PLoS One, vol. 8, no. 2, article e54831, 2013. 


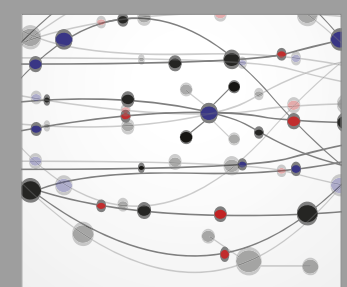

The Scientific World Journal
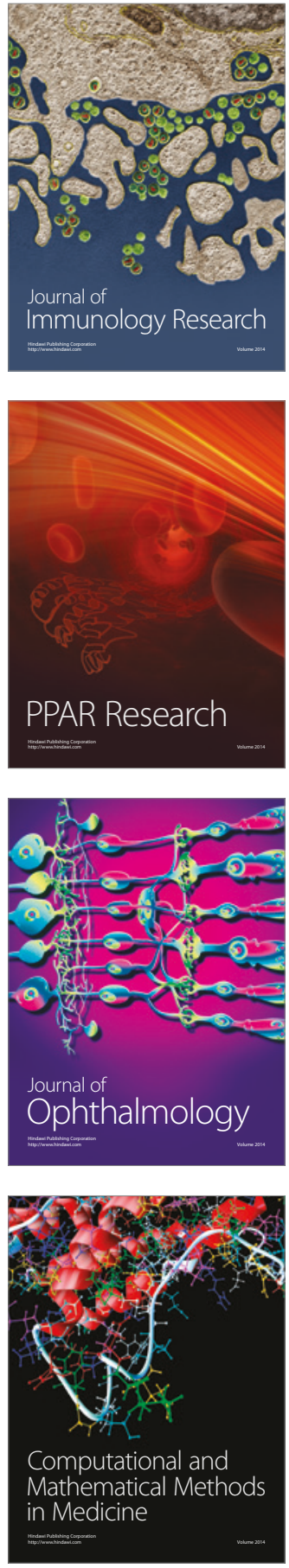

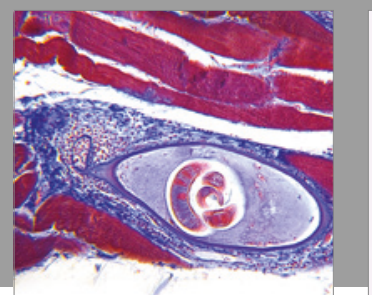

Gastroenterology Research and Practice
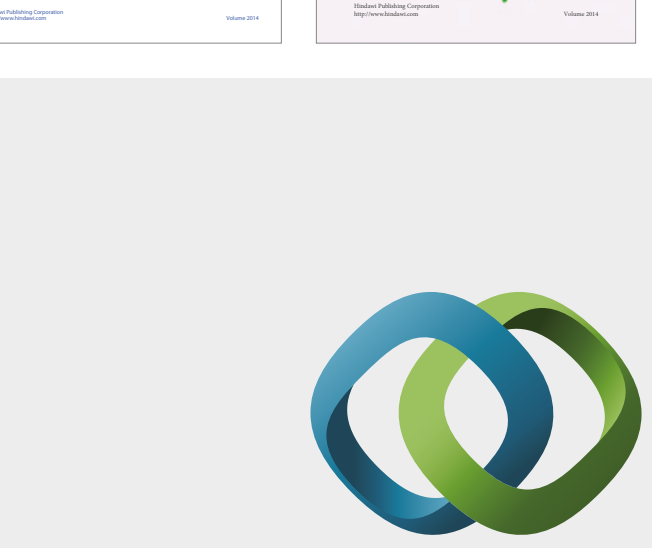

\section{Hindawi}

Submit your manuscripts at

https://www.hindawi.com
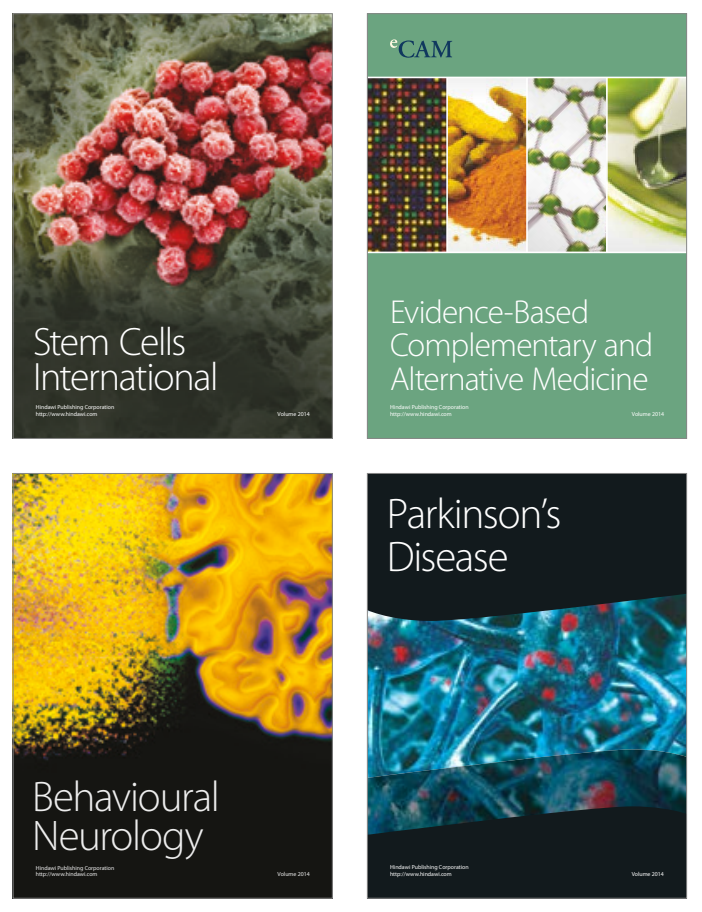
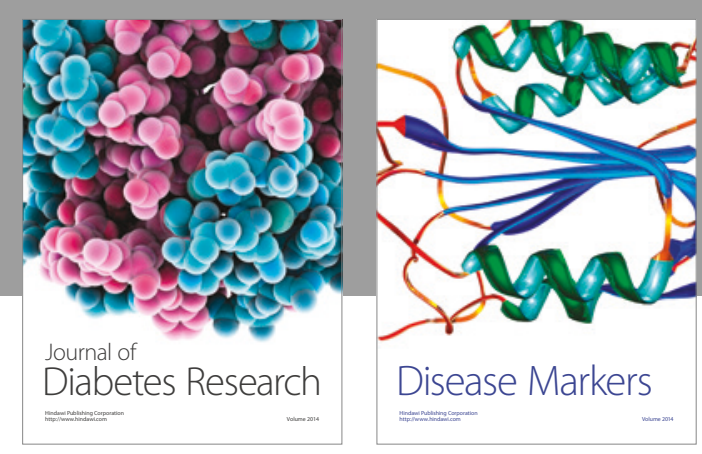

Disease Markers
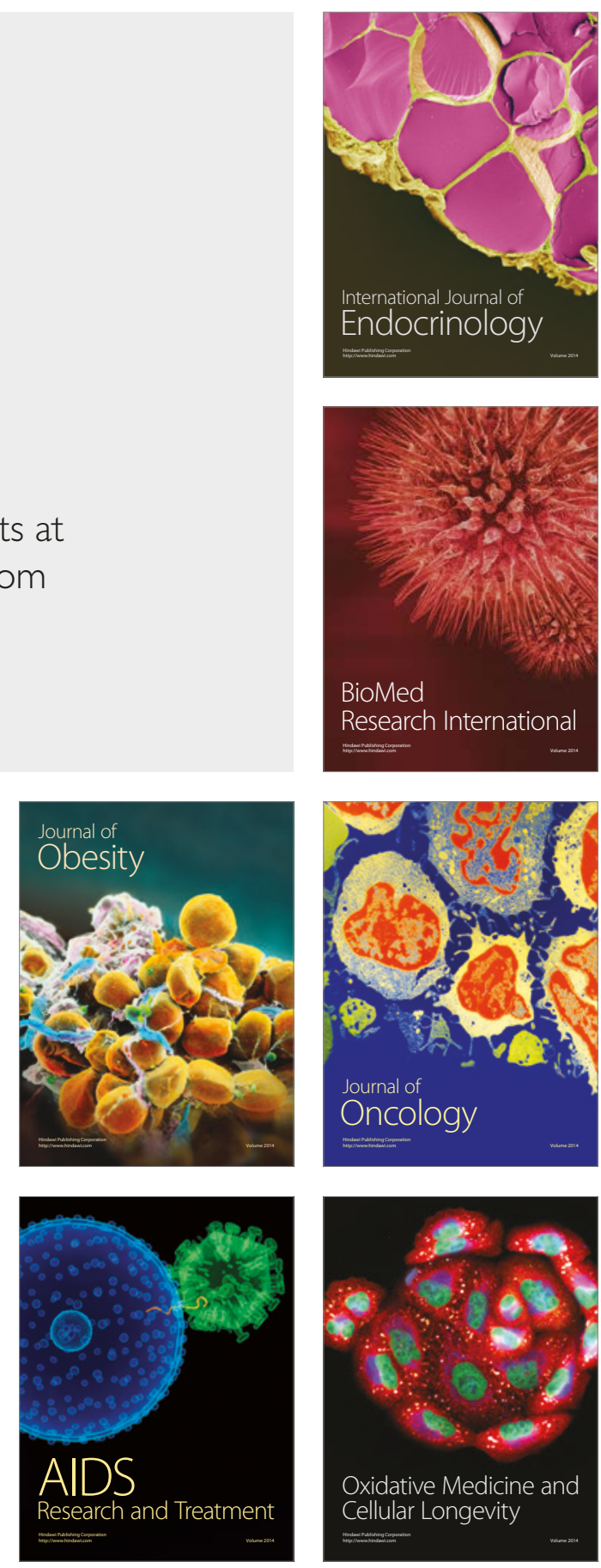